\title{
High expression of a-synuclein in damaged mitochondria with PLA2G6 dysfunction
}

\author{
Hisae Sumi-Akamaru1*, Goichi Beck1, Koei Shinzawa², Shinsuke Kato ${ }^{3}$, Yuichi Riku ${ }^{4,5}$, Mari Yoshida ${ }^{5}$, \\ Harutoshi Fujimura ${ }^{6}$, Yoshihide Tsujimoto ${ }^{2,7}$, Saburo Sakoda ${ }^{6}$ and Hideki Mochizuki ${ }^{1 *}$
}

\begin{abstract}
To clarify the role of a-synuclein (aSyn) in neuronal membrane remodeling, we analyzed the expression of aSyn in neurons with a dysfunction of PLA2G6, which is indispensable for membrane remodeling. aSyn/phosphorylated-aSyn (PaSyn) distribution and neurodegeneration were quantitatively estimated in PLA2G6-knockout (KO) mice, which demonstrate marked mitochondrial membrane degeneration. We also assessed the relationship between aSyn deposits and mitochondria in brain tissue from patients with PLA2G6-associated neurodegeneration (PLAN) and Parkinson's disease (PD), and quantitatively examined Lewy bodies (LBs) and neurons. The expression level of aSyn was elevated in PLA2G6-knockdown cells and KO mouse neurons. Strong PaSyn expression was observed in neuronal granules in $\mathrm{KO}$ mice before onset of motor symptoms. The granules were mitochondrial outer membrane protein (TOM20)-positive. Ultramicroscopy revealed that PaSyn-positive granules were localized to mitochondria with degenerated inner membranes. After symptom onset, TOM20-positive granules were frequently found in ubiquitinated spheroids, where PaSyn expression was low. Axons were atrophic, but the neuronal loss was not evident in KO mice. In PLAN neurons, small PaSyn-positive inclusions with a TOM20-positive edge were frequently observed and clustered into LBs. The surfaces of most LBs were TOM20-positive in PLAN and TOM20-negative in PD brains. The high proportion of LB-bearing neurons and the preserved neuronal number in PLAN suggested long-term survival of LB-bearing neurons. Elevated expression of aSyn/PaSyn in mitochondria appears to be the early response to PLA2G6-deficiency in neurons. The strong affinity of aSyn for damaged mitochondrial membranes may promote membrane stabilization of mitochondria and neuronal survival in neurons.
\end{abstract}

Keywords: PLA2G6, a-synuclein, Mitochondrial membrane, Lewy body

\section{Introduction}

It is well known that $\alpha$-synuclein ( $\alpha$ Syn) is a pathological marker of Parkinson disease (PD), because $\alpha$ Syn/phosphorylated $\alpha$ Syn (PaSyn) is a main component of Lewy bodies (LBs). The physiological function of $\alpha$ Syn has recently become known. As a pre-synaptic chaperone, $\alpha$ Syn promotes soluble NSF-attachment protein receptor (SNARE)-complex assembly $[9,22]$. $\alpha$ Syn also localizes to nuclei and subcellular organelles, including mitochondria and mitochondrion-associated endoplasmic reticulum (ER) membranes $[5,16,37]$. $\alpha$ Syn binds to lipid membranes $[42,43]$, in particular, to membranes with high

\footnotetext{
* Correspondence: hasumi@neurol.med.osaka-u.ac.jp; hmochizuki@neurol. med.osaka-u.ac.jp

1 Department of Neurology, Graduate School of Medicine, Osaka University, 2-2 Yamadaoka, Suita 565-0871, Japan

Full list of author information is available at the end of the article
}

curvature, such as synaptic vesicles and mitochondrial inner membranes $[5,25,52]$. The $\mathrm{N}$-terminus of $\alpha$ Syn lies along the surface of the membrane [5, 43], where it senses lipid-packing defects and leads to membrane remodeling and stabilization [7, 29].

Mitochondria comprise an inner and an outer membrane that separate the intermembrane space and the matrix. Mitochondria have various functions, including oxidative phosphorylation, lipid metabolism, endocytosis [24], apoptosis, and calcium and iron homeostasis [32]. A mitochondrial inner membrane-specific phospholipid, cardiolipin, is crucial for the integrity and function of mitochondria [25, 32]. In the brains of mice lacking $\alpha$ Syn, the mitochondrial lipid composition changes, and complex I/III activity is reduced [12]. Moreover, the Nterminus of $\alpha$ Syn regulates mitochondrial membrane 
permeability [40]. Together, these findings suggest that $\alpha$ Syn is integral to maintaining mitochondrial function.

Calcium-independent phospholipase $\mathrm{A}_{2} \beta$, encoded by PLA2G6, has diverse functions, such as releasing lipid mediators, inflammation, vascular relaxation, and secretion, by hydrolyzing the $s n-2$ ester bond in phospholipids $[8,10,27$, 28, 51]. In PLA2G6-associated neurodegeneration (PLAN) $[19,26]$, formerly called Seitelberger disease, a variety of neurological deficits are present from infancy, suggesting the importance of PLA2G6 in the brain. PLAN encompasses a number of phenotypes, such as infantile neuroaxonal dystrophy and adult-onset dystonia-parkinsonism (Park14). Although these phenotypes differ in the degree and distribution of neurodegeneration, $\alpha$ Syn/Lewy-related pathology is commonly observed $[15,31,36]$.

The pathogenesis of PLA2G6 deficiency is thought to involve dysfunction of mitochondria and membrane remodeling [13, 20]. Pla2g6-knockout (KO) mice show slow progression of motor deficits, and there is a progressive formation of spheroids and tubulovesicular structures $[23,41]$, similar to that seen in PLAN $[11,17]$. Recently, we reported that the spinal cord neurons in Pla2g6-KO mice have ultra-microscopically abnormal mitochondria, with degenerated inner membranes, which are periodic acid Schiff (PAS)-positive, negative for an inner membrane protein (cytochrome c oxidase, $\mathrm{CCO}$ ), and positive for an outer membrane protein (translocase of the outer mitochondrial membrane, TOM20) on immunohistochemistry [4, 45]. In mass spectrometry, the content of mitochondria-specific phospholipid, cardiolipin, is high in Pla2g6-KO mice. These findings suggest dysregulation of phospholipid metabolism in the mitochondrial inner membrane. We also previously demonstrated low expression of $\mathrm{CCO}$ and low production of ATP in PLA2G6-knockdown cells, suggesting mitochondrial dysfunction [3].

Genetic mutation of SNCA [34] and multiple copies of SNCA [38] cause PD, suggesting the toxic function due to a genetic abnormality of $\alpha$ Syn $[5,48]$. On the other hand, in the brains of elderly people without neuropsychiatric symptoms, where widespread $\alpha$ Syn/Lewy-related pathology is seen [39], the severity of $\alpha$ Syn/Lewy-related pathology is not associated with the clinical course in PD [14, 18]. In spite of many intensive researches $[1,5,14,18,35,48]$, the biological significance of LBs in sporadic PD and other familial PD is not yet fully understood. In this study, we aimed to clarify the reason for $\alpha$ Syn accumulation in neurons, and pathologically analyzed the relationship between $\alpha$ Syn and mitochondrial membranes in PLAN and in Pla2g6-KO mice.

\section{Materials and methods}

\section{Generation of Pla2g6-Kd cells}

We generated PLA2G6 gene knockdown (Kd) SH-SY5Y human neuroblastoma cells, as described before [3].
Briefly, SH-SY5Y neuroblastoma cell line was obtained from American Tissue Culture Collection (ATCC, Manassas, VA). Cells were grown in Dulbecco's modified Eagle's medium high glucose (high-glucose formulation, Nacalai Tesque, Kyoto, Japan) supplemented with $10 \%$ fetal bovine serum, 100 units/ml penicillin, and $100 \mu \mathrm{g} /$ $\mathrm{ml}$ streptomycin. Cell cultures were all kept at $37{ }^{\circ} \mathrm{C}$. The small interfering RNA (siRNA) targeting human PLA2G6 gene (Life technologies, Carlsbad, CA) and negative control siRNA (Qiagen, Hilden, Germany), were obtained. Subconfluent SH-SY5Y cells were transfected with siRNAs using Lipofectamine RNAiMax (Invitrogen, Carlsbad, CA). The targeting sense sequence for human PLA2G6 in SH-SY5Y cells is 5'-GACCAAAGAGCAAG UGACAAAUGUU-3'.

\section{RNA expression analysis}

The absence of the PLA2G6 expression was confirmed in Pla2g6-Kd cells, as described before [3]. Briefly, total RNA was extracted from siRNA-transfected SH-SY5Y cells using the RNeasy Kit (Qiagen, Hilden, Germany), and the RNA concentrations were determined spectrophotometrically. cDNA was generated using the SuperScript VILO cDNA Synthesis Kit (Invitrogen, Carlsbad, CA) from $100 \mathrm{ng}$ of each RNA sample. RT-PCR was used to confirm reduced expression levels of PLA2G6 gene (data not shown).

\section{Western blotting}

Cells were collected after transfection for $48 \mathrm{~h}$. Samples ( $n=6$ per group) were prepared as described before [3] Cells, which were transfected with negative control siRNA, were used as control. The protein $(10 \mu \mathrm{g})$ was separated on $15 \%$ SDS-PAGE, electrotransferred to a polyvinylidene difluoride (PVDF) membrane (Bio-Rad, CA, USA), blocked with $5 \%$ nonfat milk and incubated overnight at $4{ }^{\circ} \mathrm{C}$ with the primary antibody against GAPDH (1:1000, Millipore) and $\alpha$ synuclein ( $\alpha$ Syn, 1:200, IBL, Fujioka, Japan). The bands were visualized with enhanced chemiluminescence's reagents and exposed to X-ray film.

\section{Immunocytochemistry}

Cells were fixed in $4 \%$ paraformaldehyde for $30 \mathrm{~min}$. After washing with PBS three times, cells were permeabilized with $0.2 \%$ Triton X-100 for $30 \mathrm{~min}$, and incubated with $10 \%$ skim milk in PBS for $60 \mathrm{~min}$. The antibodies against $20-\mathrm{kDa}$ translocase of the outer mitochondrial membrane (1:100 dilution, TOM20, import receptor, expressed on the mitochondrial outer membrane; Santa Cruz, Dallas, TX) and $\alpha$ Syn (1:100 dilution, LB509, Abcam, Cambridge, England) were used as primary antibodies. Alexa Fluor1488 goat antirabbit IgG $(\mathrm{H}+\mathrm{L})$ antibody (Life Technologies) and Alexa Fluor1568 goat anti-mouse IgG $(\mathrm{H}+\mathrm{L})$ antibody 
(Life Technologies) were used as the secondary antibodies. Confocal laser-scanned images were obtained using an LSM 510 META (Carl Zeiss, Oberkochen, Germany).

\section{Animals}

All animals were handled in accordance with the Guidelines for Animal Experimentation of Osaka University (No.26-044-000).

Mice carried a homozygous disruption of Pla2g6 on a C57BL/6 background [41], aged 15 weeks ( $n=3$, preclinical stage, 1 male and 2 females); 1 year $(n=4$, early symptomatic stage, 1 male and 3 females); and 2 years ( $n=5$, end stage, 2 males and 3 females); and wild-type mice, aged 15 weeks ( $n=3,1$ male and 2 females); and 2 years ( $n=4,1$ male and 3 females) were obtained. After perfusion of $4 \%$ paraformaldehyde, spinal cords were removed and immersed in the same fixative overnight at $4{ }^{\circ} \mathrm{C}$, after which 4 - $\mu \mathrm{m}$-thick paraffin sections were prepared. Sciatic nerves were also obtained. The sciatic nerves and small pieces of the spinal cord were fixed with $2.5 \%$ glutaraldehyde and processed to epon blocks, as described previously [44]. Epon sections (1- $\mu \mathrm{m}$-thick) were stained with thionine and PAS.

\section{Autopsy}

The research presented in this study has been approved by the University Ethics Committee Osaka University Graduate School of Medicine (No. 10038). Consent for autopsies were obtained from legal representatives for all subjects in accordance with local institutional review board requirements, which was approved by the University Ethics Committee (Osaka University Graduate School of Medicine, Osaka, Japan).

Autopsy samples of one PLAN case (age at death: 20 years; disease duration: 17 years), seven Parkinson's disease cases (age at death: $73 \pm 9$ years; disease duration: $13 \pm 8$ years), and five non-neurodegenerative control cases (age at death: $61 \pm 16$ years) were obtained. Paraffin-embedded 6 - $\mu \mathrm{m}$-thick sections of the midbrain, at the level of the red nucleus, upper pons, and upper medulla, were prepared and examined, because of the high frequency of LBs in those area in both PLAN [15, 31, 36] and PD.

\section{Immunohistochemistry}

Deparaffinized sections were incubated for $30 \mathrm{~min}$ with $0.3 \% \mathrm{H}_{2} \mathrm{O}_{2}$ to quench endogenous peroxidase activity, and were then washed with phosphate-buffered saline (PBS). The primary antibodies used were rabbit polyclonal antibodies against $\alpha$-Syn (1:2000 dilution for mouse, 1:5000 dilution for human; Sigma - Aldrich, St. Louis, MO), TOM20 (1:40 dilution for mouse, 1:100 dilution for human, Santa Cruz), cathepsin D (lysosomal enzyme, 1:400 dilution, Dako, Glostrup, Denmark), ubiquitin (1:2000 dilution, DAKO), and mouse monoclonal antibodies against P $\alpha$ Syn (specific for Ser129phosphortlated $\alpha$ Syn, 1:800 dilution for mouse, 1:2000 dilution for human, pSyn\#64; Wako, Osaka, Japan) and cytochrome $c$ oxidase subunit IV $(\mathrm{CCO}$, respiratory complex IV, expressed on the mitochondrial inner membrane; 1:300 dilution for mouse, 1: 1000 dilution for human; Invitrogen) and KDEL (Lys-Asp-Glu-Leu, 1:500 dilution, Enzo Life Sciences, Farmingdale, NY). Goat anti-rabbit and anti-mouse immunoglobulins conjugated to peroxidase-labeled dextran polymer (Dako Envision+, Dako) were used as secondary antibodies. Reaction products were visualized with 3,3'-diaminobenzidine tetrahydrochloride (Vector Laboratories, Burlingame, CA), and hematoxylin was used to counterstain the cell nuclei. The immunostaining patterns were compared in serial sections. Some sections were additionally stained with Luxol Fast Blue (LFB) or PAS.

\section{Double immunohistochemistry}

For double immunohistochemistry, two primary antibodies were combined, including antibodies for $\alpha$ Syn ( $\alpha$ Syn or P $\alpha$ Syn), mitochondrial membrane markers (CCO or TOM20), ubiquitin, and tyrosine hydroxylase (TH). The VECTASTAIN ABC-AP kit (Vector Laboratories) and ALKALINE PHOSPHATASE SUBSTRATE KIT IV BCIP/NBT (Vector Laboratories) were used for the secondary antibody and visualization of reaction products, respectively.

\section{Quantitative pathological analysis of anterior horn cells and sciatic nerves of mice}

We estimated the number of neurons filled with P $\alpha$ Synpositive granules and the number of motor neurons in the anterior part of mouse cervical spinal cord and myelinated fibers in sciatic nerves. The neurons with clear nucleoli and cell bodies with a diameter greater than $25 \mu \mathrm{m}$, presumed to be alpha motor neurons, were counted [44]. To this end, video images were obtained for each 4- $\mu \mathrm{m}$-thick Nissl-stained paraffin section and each 1- $\mu \mathrm{m}$-thick toluidine blue-stained epon section, using a digital camera (KEYENCE VB-7010, KEYENCE, Osaka, Japan) attached to a light microscope (ECLIPSEE800, Nikon, Tokyo, Japan). The diameters of motoneurons showing clear nucleoli and cell bodies, and the myelinated fibers in the sciatic nerves were measured using image analysis software (VH-H1A5, KEYENCE). Four sections of cervical cords were examined for each mouse. For the sciatic nerves, three fields $(100 \times$ magnification) per mouse were examined. The number of motoneurons, large myelinated fibers (diameter, $>10 \mu \mathrm{m}$ ), and total myelinated fibers in wild-type mice (2-years-old) and Pla2g6-KO mice (1-year-old and 2-years-old) was counted. The density of myelinated fibers $\left(\right.$ per $\mathrm{mm}^{2}$ ) was 
calculated for each mouse. The differences in the number of motoneurons and the fiber density between wild-type mice and Pla2g6-KO mice were statistically analyzed using the Wilcoxon rank sum test.

\section{Ultrastructural analysis}

Ultrathin sections of the spinal cord from Pla2G6-KO mice aged 15 weeks, 56 weeks, or 100 weeks were prepared and stained with uranyl acetate and lead citrate, and examined using a transmission electron microscope (H-7650; Hitachi High-Technologies Co., Tokyo, Japan).

\section{Quantitative pathological analysis of TOM20-expression} on the surface of human LBs

Double immunohistochemistry was performed for P $\alpha$ Syn (in brown) and for TOM20 (in blue) in the substantia nigra, in the locus coeruleus, or in the dorsal motor nuclei of the vagus nerve. Neuronal round or spherical P $\alpha$ Syn-positive inclusions with a clear contour were defined as LBs. In double immunohistochemistry, P $\alpha$ Synpositive LBs (brown) were separated into three types, according to the expression pattern of TOM20 (blue) on the surface: TOM20-negative, partially TOM20-positive, and completely TOM20-positive (Fig. 7). Where less than half of the LB surface was positive for TOM20, the LB was classified as "partially TOM20-positive" and where more than half of the LB surface was positive for TOM20, the LB was classified as "completely TOM20positive". We counted the number of each type of LB in the substantia nigra, the locus coeruleus, and dorsal motor nuclei of the vagus in each patient. Slides were examined with $\mathrm{a} \times 40$ objective, and the microscope images were viewed on video-screen. The contours of the substantia nigra and the locus coeruleus, and dorsal motor nuclei of the vagus were drawn on the slide. The number of LBs with various surface expression levels of TOM20 was compared between PLAN and PD sections.

\section{Estimation of dopaminergic neuron density and the proportion of LB-bearing neurons in controls and PD and PLAN patients}

Double immunohistochemistry was performed against PaSyn (brown) and TH (blue) in human brainstem sections. In double immunohistochemistry, we counted the number of TH-positive (dopaminergic) neurons bearing LBs and total TH-positive neurons in the substantia nigra and the locus coeruleus [47]. Slides were examined with $\mathrm{a} \times 40$ objective, and the microscope image was viewed on a video-screen. The contours of the substantia nigra and the locus coeruleus were drawn on the slide. The neuron density over three fields was calculated for each patient. The density (per $\mathrm{mm}^{2}$ ) of $\mathrm{TH}$-positive neurons bearing LBs and total $\mathrm{TH}$-positive neurons were compared between PLAN, PD $(n=7)$, and control $(n=5)$ individuals.

\section{Estimation of the influence of LBs on TH expression levels in dopaminergic neurons}

Double immunohistochemistry was performed for P $\alpha$ Syn (brown) and TH (blue). The immunostaining of $\mathrm{TH}$ was compared between dopaminergic neurons with LBs and those without LBs, in the substantia nigra and locus coeruleus. The brainstem was compared between PLAN and PD $(n=7)$ individuals.

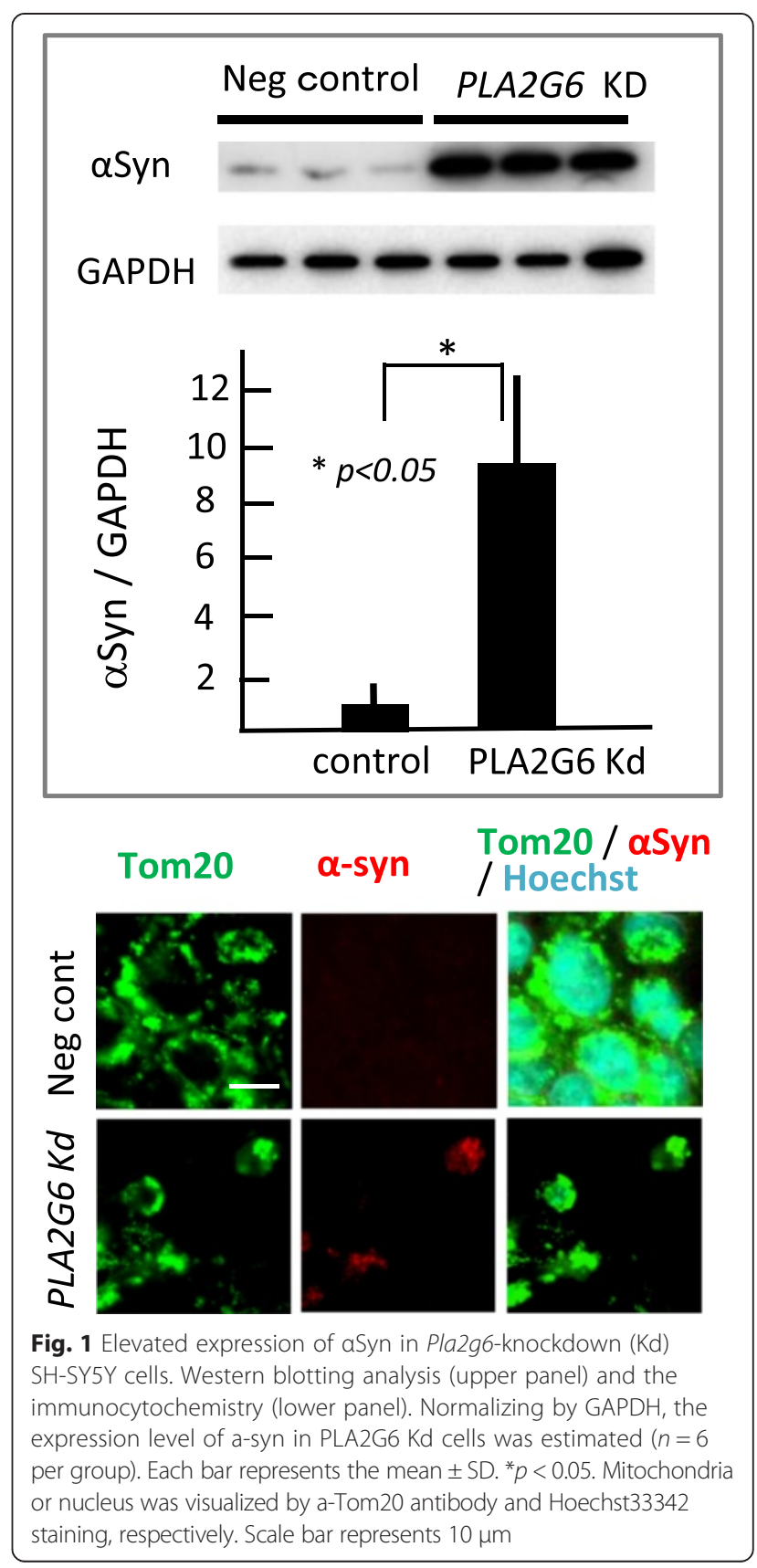




\section{Statistical analysis}

All statistical analyses of histopathological data in mice were performed using SPSS Statistics software for Windows version 20.0 (SPSS, Chicago, IL). Data are expressed as the mean (SD). Mann-Whitney $U$ test was used to analyze the ratio of $\alpha \mathrm{Syn} / \mathrm{GADPH}$ in cultured cells, the number of neurons and the density of large myelinated fibers and total myelinated fibers in mice. $P$ values of less than 0.05 were considered as statistically significant.
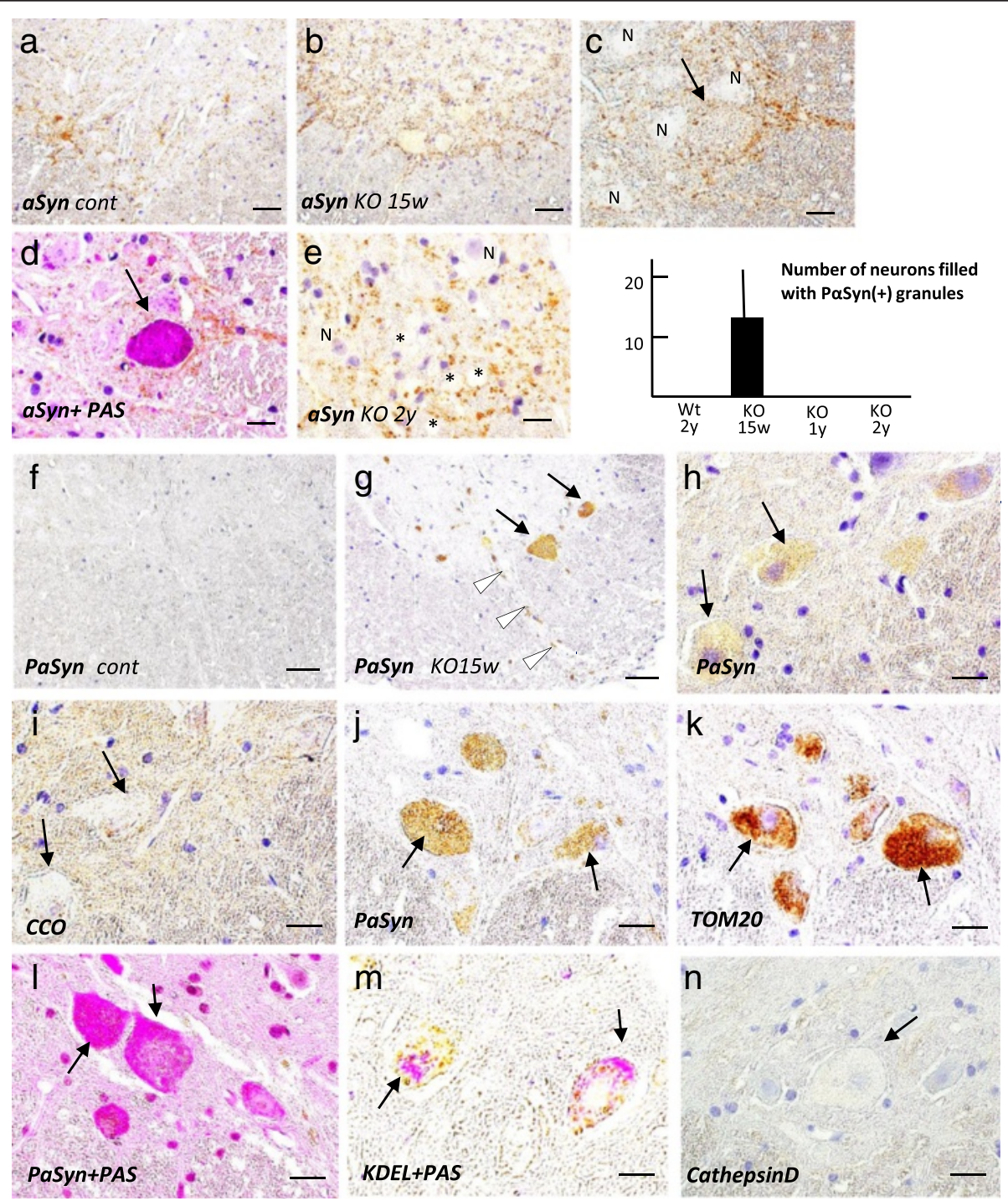

Fig. 2 Prominent accumulation ofaSyn/phosphorylated aSyn (PaSyn) in the spinal cord of Pla2g6-knockout (KO) mice at the preclinical-stage. a, $\mathbf{f}$ wildtype mice; b-d, g-m, Pla2g6-KO mice at 15 weeks; e, Pla2g6-KO mice at 2 years; a-n, Anterior part of spinal cords; a-c, e, Immunohistochemistry for aSyn; $\mathbf{d}$, Immunohistochemistry for aSyn plus Periodic Acid Shiff (PAS); $\mathbf{f - n}$, Immunohistochemistry against PaSyn (f-h, j), CCO (i), and TOM20 (k), and cathepsin D (n), Immunohistochemistry for PaSyn (I) or KDEL (m) plus PAS, respectively; Scale bar represents $80 \mu \mathrm{m}$ in $(\mathbf{a}, \mathbf{b}, \mathbf{f}, \mathbf{g})$, and $20 \mu \mathrm{m}$ in $(\mathbf{c}-\mathbf{e}, \mathbf{h}-\mathbf{n})$. The graph shows the number of neurons filled with PaSyn-positive granules in wild-type mice at 2 years of and Pla2g6-KO mice at 15 weeks, 1 year, and 2 years of age. $\mathbf{a}$ The gray matter of the control spinal cord is moderately stained, showing a punctate pattern; $\mathbf{b}, \mathbf{c}$ aSyn expression is highly diffuse in the gray matter of young Pla2g6-KO mice. The neuron is mildly positive for aSyn (arrow in c), which is filled with PAS-positive granules, as shown in a serial section (d). e In the gray matter of KO mice at end stage, several vacuoles and spheroids are seen, which are negative for aSyn $\left(^{*}\right)$. $\mathbf{f}$ No staining is seen in the control. (g) In KO mice at the pre-clinical stage, there are many tiny PaSyn-positive granules in the anterior horn cells (arrows) and the proximal axons (white arrowheads). h, i, PaSyn-positive small granules (arrows in h) are virtually negative for CCO (arrows in i) in the same neurons as in the serial section. $\mathbf{j}, \mathbf{k}$ Very small PaSyn-positive granules (arrows in $\mathbf{j}$ ) are prominently observed in the same neurons filled with TOM20-positive granules (arrows in $\mathbf{k}$ ) in the serial section. I, $\mathbf{m}$, PAS-positive granules are PaSyn-positive (I, arrows) and KDEL-negative ( $\mathbf{m}$, arrows). $\mathbf{n}$ A swollen neuron is negative for cathepsin $\mathrm{D}$ (arrow) 


\section{Results}

\section{Cultured cells}

\section{High expression of aSyn in Pla2g6-knockdown cells}

To clarify the relationship between $\alpha$ Syn and PLA2G6 dysfunction in cultured neurons, we analyzed the expression level of $\alpha$ Syn in Pla2g6-Kd SH-SY5Y human neuroblastoma cells. In the western blotting analysis of Pla2g6-Kd cells, $\alpha$ Syn expression was strikingly high (Fig. 1). The difference between $\mathrm{Kd}$ cells and negative control cells was significant $(n=6$ per group, $p<0.05$ ). Colocalization of $\alpha$ Syn and translocator of outer mitochondrial membrane 20 (TOM20) was demonstrated by immunocytochemistry.

\section{Mice}

Strong expression of aSyn in the spinal cord of Pla2g6-KO mice at the preclinical-stage

To clarify the relationship between $\alpha$ Syn and PLA2G6 dysfunction in mice in vivo, we analyzed the distribution of $\alpha$ Syn in Pla2g6-KO mice using immunohistochemistry. In wild-type mice of both 15 weeks and 2 years of age, the neuropil was moderately stained for $\alpha$ Syn, with punctate pattern (Fig. 2a). In Pla2g6-KO mice at the pre-clinical stage (15 weeks), the immunoreactivity of $\alpha$ Syn was highly diffuse in the gray matter (Fig. 2b). $\alpha$ Syn staining in the neuropil had a punctate pattern, similar to that in wild-type mice, but the cytoplasm of some swollen neurons was also mildly positive for $\alpha$ Syn (Fig. 2c). PAS staining showed that $\alpha$ Syn-positive swollen neurons were filled with PAS-positive granules (Fig. 2d). With age, large vacuoles and spheroids in the neuropil were increased in number, where the expression level of $\alpha$ Syn was almost none or low (Fig. 2e).

\section{Strong expression of PaSyn in CCO-negative, TOM20- positive granules in young Pla2g6-KO mice}

To determine if there was a relationship between subcellular micro-organelle localization and accumulation of P $\alpha$ Syn, the most important modified-form of $\alpha$ Syn after transcription, we compared the distribution of
P $\alpha$ Syn with markers of subcellular micro-organelles in neurons of Pla2g6-KO mice. Immunohistochemistry with an antibody specifically directed against Ser129phosphorylated $\alpha$ Syn (pSyn\#64) revealed the absence of PaSyn in the wild-type mice (Fig. 2f). In Pla2g6-KO mice at 15 weeks, very small PaSyn-positive granules were prominent in the cytoplasm and proximal axons of anterior horn cells (Fig. 2g, h, j) and dorsal root ganglion cells (data not shown). In some of the swollen neurons filled with small $\mathrm{P} \alpha \mathrm{Syn}$-positive granules, the nuclei were atrophic (Fig. 2h, j). By comparing serial sections, we found that the neurons filled with PoSynpositive granules were CCO-negative (Fig. 2i) and TOM20-positive (Fig. 2k). The granules were PASpositive (Fig. 2l), KDEL-negative (Fig. 2m), and cathepsin D-negative (Fig. 2n). The number of neurons filled with $\mathrm{P} \alpha$ Syn-positive granules is shown in the graph (Fig. 2) and Table 1.

\section{Morphological changes of PaSyn-positive granules in Pla2g6-KO mice with age}

To assess the relationships between high P $\alpha$ Syn expression and neurodegeneration in Pla2g6-KO mice, we analyzed the age-dependent distribution of PaSyn. In Pla2g6-KO mice at 15 weeks, very small PoSyn-positive granules were prominently observed in the proximal axons (Fig. 3a) and cytoplasm of anterior horn cells. At the early symptomatic stage ( 1 year), there were only a few PoSyn-positive granules in neuronal cytoplasm. Swollen neurons, filled with $\mathrm{P} \alpha \mathrm{Syn}$-positive granules, were completely absent (Table 1 ). In the proximal axons, there were many $\mathrm{P} \alpha \mathrm{Syn}$-positive granules, some of which were large and deformed (Fig. 3b). Double immunohistochemistry revealed $\mathrm{P} \alpha$ Syn co-localization with TOM20 in the granules (Fig. 3c).

At the end stage (2 years), some of the P $\alpha$ Syn-positive granules showed degenerated membranes (Fig. 3d, e). Using double immunohistochemistry, P $\alpha$ Syn was shown to co-localize with TOM20 in the granules and the degenerated membrane of the granules (Fig. 3f).

Table 1 The time course of Pa-syn expression in neurons and the number of motor neurons and myelinated fibers of Pla2g6-knockout (KO) mice

\begin{tabular}{|c|c|c|c|c|c|}
\hline & & \multicolumn{2}{|l|}{ Wt } & \multicolumn{2}{|l|}{$\mathrm{KO}$} \\
\hline & & $2 y(n=4)$ & $15 \mathrm{w}(n=3)$ & $1 \mathrm{y}(n=4)$ & $2 y(n=5)$ \\
\hline $\begin{array}{l}\text { Number of neurons filled } \\
\text { with PaSyn }(+) \text { granules }\end{array}$ & & 0 & $12(10)$ & 0 & 0 \\
\hline Number of $\mathrm{AHCs}$ & & $44(6)$ & n.e. & $44(2)$ & $33(10)$ \\
\hline Sciatic nerve (distal axon) & Density of myelinated fibers & $17567(1513)$ & n.e. & $14281(2086)$ & $18850(1745)$ \\
\hline Sciatic nerve (distal axon) & Density of large myelinated fibers & $1623(314)$ & n.e. & $613(172)$ & $156^{*}(104)$ \\
\hline
\end{tabular}

PaSyn phosphorylated a-synuclein, Wt wild-type, KO Pla2g6-knockout, $15 \mathrm{w} 15$ weeks, 1 y 1 year, 2 y 2 years, AHC anterior horn cell, number of anterior horn cells mean (SD), n.e. not examined, Density of myelinated fibers number of myelinated fibers per $\mathrm{mm}^{2}$, large myelinated fibers myelinated fibers with a diameter larger than $10 \mu \mathrm{m},{ }^{*} p<0.05$ 

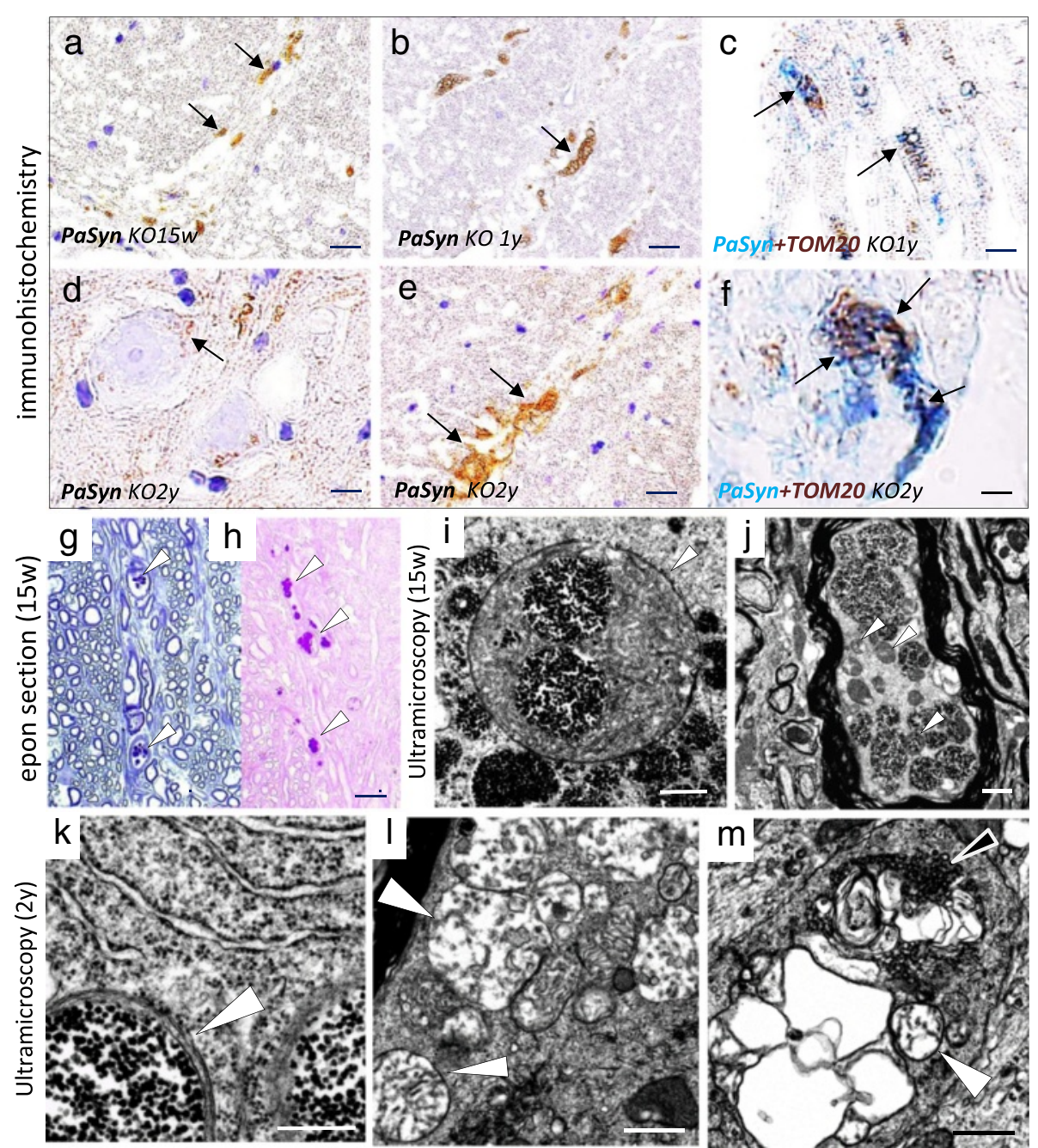

Fig. 3 Age-dependent morphological changes in PaSyn-positive mitochondria in axons. a-f paraffin section; $\mathbf{g}$, $\mathbf{h}$ epon semi-thin section; $\mathbf{i}-\mathbf{m}$ ultramicroscopy; a, g-j Pla2g6-KO mouse at 15 weeks; (b, c) Pla2g6-KO mice at 1 year; $\mathbf{d}-\mathbf{f}, \mathbf{k}-\mathbf{m}$, Pla2g6-KO mice at the 2 years. a, b, e, g, h, j, l, $\mathbf{m}$ Anterior column of the spinal cord; $\mathbf{c}, \mathbf{f}$ anterior root; $\mathbf{d}, \mathbf{i}$, $\mathbf{k}$ perinuclear of neuron; $\mathbf{a}, \mathbf{b}, \mathbf{d}, \mathbf{e}$ Immunohistochemistry for PaSyn; $\mathbf{c}, \mathbf{f}$ double immunohistochemistry for PaSyn (blue) and TOM20 (brown); $\mathbf{g}$ thionine staining; $\mathbf{h}$ PAS staining. Scale bars represent $40 \mu \mathrm{m}$ in $(\mathbf{a}, \mathbf{b}, \mathbf{e}), 10 \mu \mathrm{m}$ in $(\mathbf{c}, \mathbf{d}, \mathbf{g}, \mathbf{h}), 5 \mu \mathrm{m}$ in $(\mathbf{f}), 500 \mathrm{~nm}$ in (i and $\mathbf{k}$ ), and $1 \mu \mathrm{m}$ in $(\mathbf{j}, \mathbf{l}, \mathbf{m})$. a At preclinical-stage, small PaSyn-positive granules (arrows) could be seen in axons. $\mathbf{b}$ At early symptomatic stage, the PaSyn-positive granules became large and irregular-shaped (arrows). c Co-localization of PaSyn and TOM20 is evident (arrows) in the granules. $\mathbf{d}$ At end stage, PaSyn-positive granules and membranes (arrow) are seen in neurons and in the neuropil. e PaSyn-positive membranous structures (arrows) coincide with the small PaSyn-positive granules. $\mathbf{f}$ The membranous structures and granules that are positive for PaSyn and TOM20 combine into a complex (arrows). $\mathbf{g}, \mathbf{h}$ In the axons, there are many dark-colored granules (arrows in $\mathbf{g}$ ), which are clearly stained by PAS (arrow in $\mathbf{h}$ ). $\mathbf{i}$ In the perinuclear space of swollen neuron filled with granules, mitochondrion with partly degenerated crista (arrowhead) and numerous round bodies filled with dense granules. $\mathbf{j}$ In a myelinated axon, many spherical mitochondria and round bodies filled with dense granules are seen. The structure of the axon is virtually normal. $\mathbf{k}$ In the mitochondrion (arrowhead) around the nuclei of neurons, most of the cristae are replaced by dense granules. I Many mitochondria with tubular and vesicular cristae (arrowheads) cluster in the axon. $\mathbf{m}$ Membranous degeneration, tubulovesicular structures (black arrowhead) and abnormal mitochondria (arrowhead) can be seen together in the spheroid

Semi-thin and ultra-microscopic image of small PaSynpositive granules in Pla2g6-KO mice

To define the structure of small $\mathrm{P} \alpha \mathrm{Syn}$-positive granules in Pla2g6-KO mouse neurons, we compared the the structures observed on semi-thin or ultra-microscopic pictures with the small P $\alpha$ Syn-positive granules seen on paraffin sections. In thionine-stained semi-thin sections, we observed dark colored granules in the cytoplasm and proximal axons of neurons in young $\mathrm{KO}$ mice (Fig. $3 \mathrm{~g}$ ). The granules were clearly PAS-positive (Fig. 3h), and showed a similar size and distribution as PaSyn-positive granules. Ultra-microscopically, round mitochondria could be seen in which the cristae were partially replaced by small dense granules both in the perinuclear space of 
neurons (Fig. 3i) and in axons (Fig. 3j). These abnormal mitochondria and round bodies were of a similar size and had a similar distribution as the PAS-positive granules in semi-thin sections, suggesting that PAS-positive granules were indicative of P $\alpha$ Syn-loading abnormal mitochondria.

In the aged $\mathrm{KO}$ mice, fewer round mitochondria could be seen in which the cristae were partially replaced by small dense granules in the perinuclear space of neurons (Fig. 3k). In the axons, mitochondria with branching and tubular cristae were frequently seen, and were clustered together (Fig. 31). In the spheroid, highly degenerated mitochondria, tubulovesicular structures and degenerated membranes were combined to form an irregular structure (Fig. 3m), suggesting severe degeneration of mitochondrial membranes.

\section{Different distribution of ubiquitin and PaSyn in Pla2g6- KO mice}

To determine whether the ubiquitin-proteasome system is activated in P $\alpha$ Syn-loading mitochondria, we estimated the relationship between ubiquitin and mitochondrial membrane protein by double immunohistochemistry. At the pre-clinical stage, there were rare ubiquitin deposits in the spinal cord of Pla2g6-KO mice (Fig. 4a-c). At the early symptomatic stage (1-year-old), ubiquitin-positive structures were frequently observed in the neuropil and the axons (Fig. 4d), but not in the neuronal cytoplasm. Double immunohistochemistry revealed that the distribution of PoSyn and ubiquitin in the axons was markedly different (Fig. 4e). At the end stage (2-year-old), ubiquitination became severe in axons, but no ubiquitination was observed
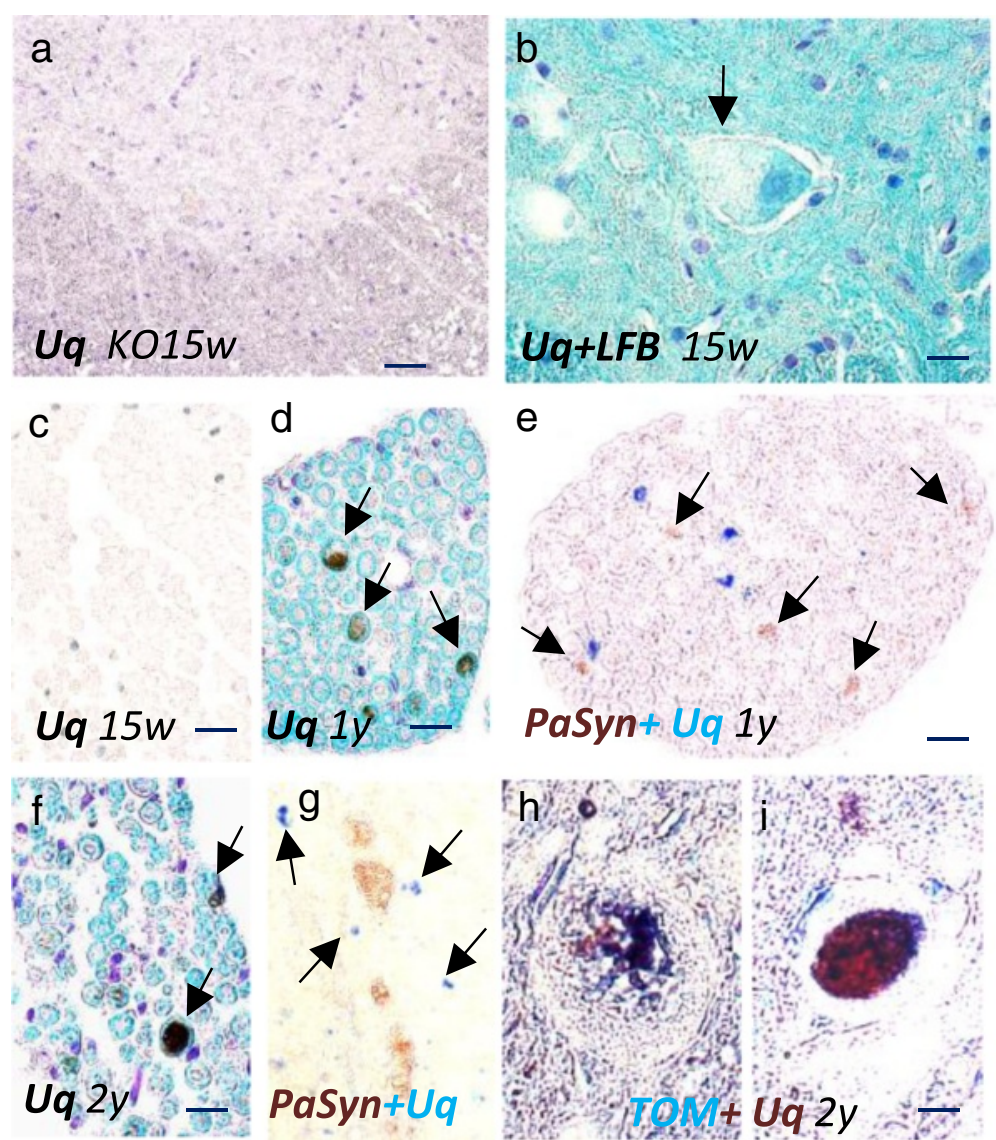

Fig. 4 Ubiquitination of mitochondrial membranes at later stages. a-c Pla2g6-KO mouse (15 w); d, e Pla2g6-KO mouse (1 year); f-i Pla2g6-KO mouse (2 years); $\mathbf{a}, \mathbf{b}, \mathbf{g}$ anterior part of the spinal cord; $\mathbf{c}-\mathbf{f}$ anterior root; $\mathbf{h}, \mathbf{i}$ posterior part of the spinal cord; $\mathbf{a}, \mathbf{c}$ immunohistochemistry for ubiquitin; b, d, f immunohistochemistry for ubiquitin plus LFB staining; e, g double immunohistochemistry for PaSyn (brown) and ubiquitin (blue); h, i double immunohistochemistry for ubiquitin (brown) and TOM20 (blue); Scale bars represent $80 \mu \mathrm{m}$ in (a), $20 \mu \mathrm{m}$ in (b-g) and $10 \mu \mathrm{m}$ in $(\mathbf{h}, \mathbf{i})$. a At the pre-clinical stage, there is almost no expression of ubiquitin. $\mathbf{b}$ The anterior horn cell (arrow), filled with small granules, is negative for ubiquitin. $\mathbf{c}$ In the anterior root, no ubiquitin-positive axon can be observed. $\mathbf{d}$ At the early symptomatic stage, ubiquitin-positive axons (arrows) with LFB-positive (blue) myelin are seen in the anterior root. e The distribution of small PaSyn-positive granules (brown, arrows) and ubiquitin (blue) are different. $\mathbf{f}$ Ubiquitin-positive axons (arrows) are shown. Most of the myelinated fibers have become atrophic. $\mathbf{g}$ The distribution of small PaSyn-positive granules (brown) and ubiquitin (blue) differs. $\mathbf{h}$ In the center of a large spheroid, TOM20-positive (blue) granules are ubiquitin-positive (brown, arrow). i In the markedly ubiquitinated spheroid (brown), the expression of TOM20 (blue) is still observed 
in the cytoplasm (data not shown). Double immunohistochemistry showed almost no colocalization of P $\alpha$ Syn and ubiquitin (Fig. 4g) but showed frequent colocalization of TOM20 and ubiquitin in the spheroids (Fig. 4h, i).

\section{Significant decrease in the density of large myelinated fibers, but not neuron number, in the spinal cord}

To clarify the degree of neurodegeneration in axons and neurons that contained $\mathrm{P} \alpha \mathrm{Syn}$-positive granules, from young mice, we estimated the number of axons and neurons in Pla2g6-KO mice after symptom onset. Axonal degeneration progressed as reported previously [41] and myelinated fibers became atrophic with age. Quantitative analysis (Table 1) revealed a significant decrease in large myelinated fibers $(>10 \mu \mathrm{m}$ in diameter) with age (KO mice $1 \mathrm{y}, n=4, p=0.057$; $\mathrm{KO}$ mice $2 \mathrm{y}, n=5, p<0.05$ ). The density of the total myelinated fibers in Pla2g6-KO mice after onset was similar to that in the wild-type mice. The number of anterior horn cells in Pla2g6-KO mice at the early symptomatic stage was similar to that of wild-type mice. Even at the end stage, the number of neurons was not significantly different between Pla2g6$\mathrm{KO}$ and wild-type mice $(p=0.067)$.

\section{Human \\ Prominent formation of Lewy Bodies (LBs) in a PLAN patient with a mutation in PLA2G6}

In the midbrain, upper pons, and upper medulla of both PD and PLAN patients, numerous neuronal inclusions that were positive for $\alpha$ Syn and P $\alpha$ Syn (pSyn\#64) could be seen. The affected areas included the substantia nigra, dorsal raphe nucleus, locus coeruleus, dorsal motor nuclei of the vagus nerve, and the reticular formation. Morphologically, the inclusions were round or spherical, similar to Pale bodies or LBs seen in PD (Fig. 5a). In PD, most of the P $\alpha$ Syn-positive LBs were present in the neuropil, particularly in the region showing severe neuronal loss. In the brain of the PLAN patient, LBs were observed both in the neurons and in the neuropil

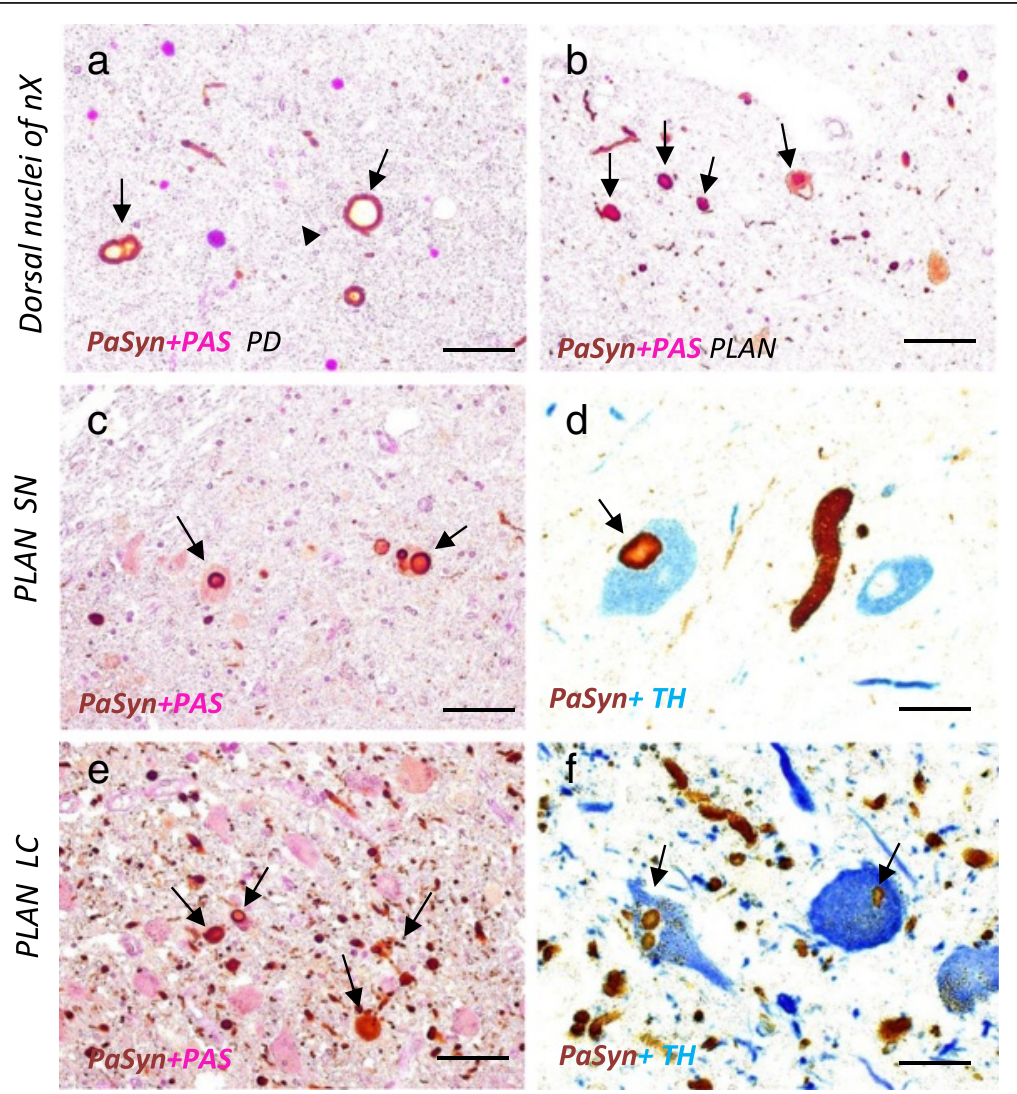

Fig. 5 Prominent formation of Lewy bodies in PLAN. a Parkinson's disease (PD) case; b-f PLA2G6-associated neurodegeneration (PLAN) case; a, b Dorsal motor nuclei of vagus (nX); c, d Locus coeruleus (LC); e, f Substantia nigra (SN); a-c, e Immunohistochemistry for PaSyn plus PAS staining; $\mathbf{d}$, f Double immunohistochemistry for PaSyn (brown) and tyrosine hydroxylase (TH, blue). Scale bars represent 40 um in (a-c, e), and $20 \mu \mathrm{m}$ in (d, f). a In PD, PaSyn-positive inclusions, Lewy bodies (arrows) are observed mainly in the neuropil. $\mathbf{b}$ In PLAN, there are many Lewy neurites and Lewy bodies ( $L B S$, arrows) both in the neurons and in the neuropil. c Cytoplasmic LBs are indicated with arrows. d Spherical Lewy neurites in the center of this panel and a LB in TH-positive neuron (arrow) are shown. $\mathbf{e}$ The neurons in the locus coeruleus are well preserved despite prominent LBs (arrows). f The LBs in TH-positive neurons are small, but have the apparent core and halo structure (arrows) 

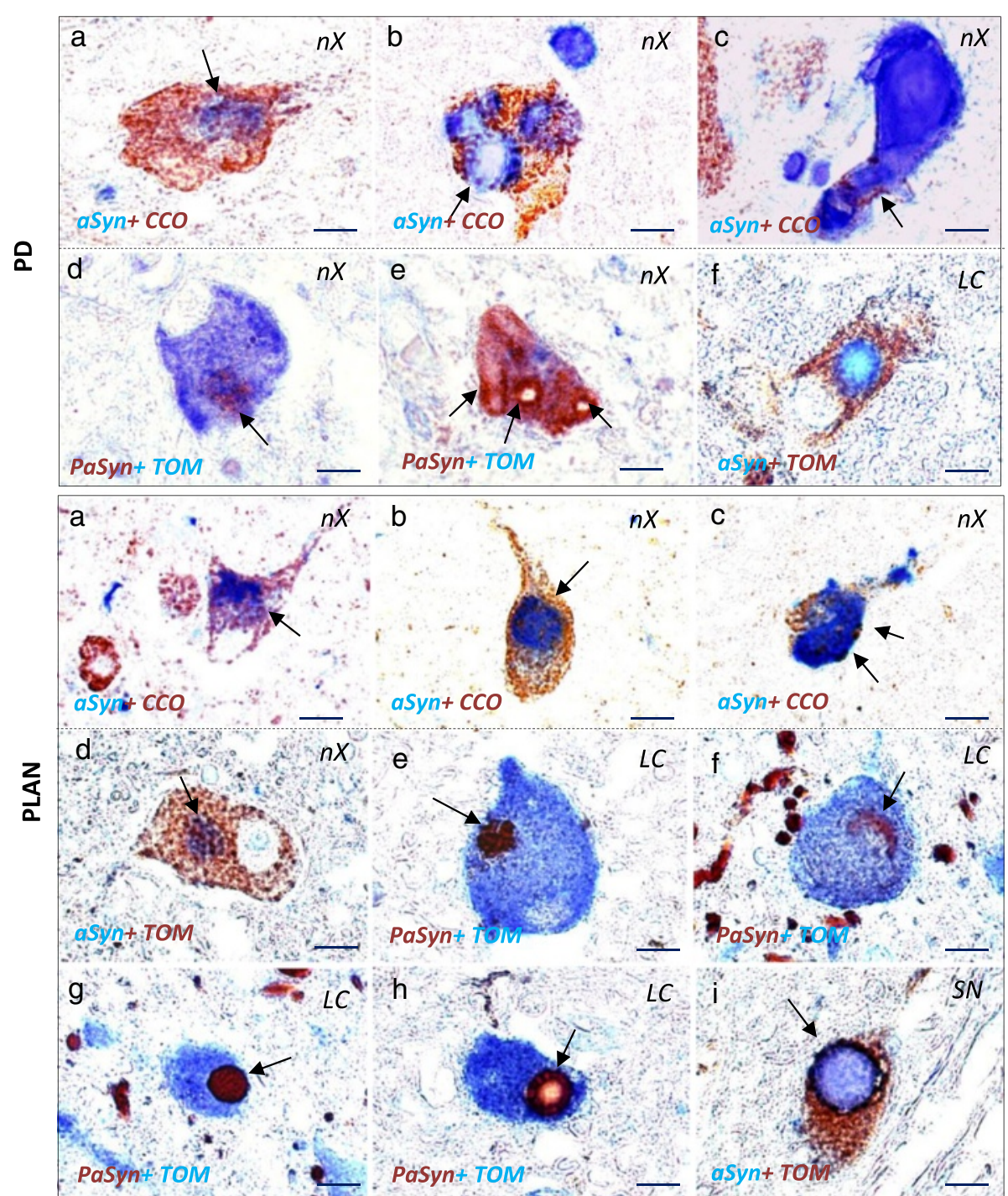

Fig. 6 The relationship between the distribution of aSyn or PaSyn and mitochondrial membrane proteins. Upper panels, Parkinson's disease (PD); a-e Dorsal motor nuclei of the vagus; $\mathbf{f}$ Locus coeruleus; a-c Double immunohistochemistry for aSyn (blue) and CCO (brown); $\mathbf{d}$, e Double immunohistochemistry for PaSyn (brown) and TOM20 (blue); f Double immunohistochemistry for aSyn (blue) and TOM20 (brown). Lower panels, PLA2G6-associated neurodegeneration (PLAN); a-d Dorsal motor nuclei of vagus; e-h Locus coeruleus; i Substantia nigra; a-c Double immunohistochemistry for aSyn (blue) and CCO (brown); d, i Double immunohistochemistry for aSyn (blue) and TOM20 (brown); e-h Double immunohistochemistry for PaSyn (brown) and TOM20 (blue). Scale bar represents $8 \mu \mathrm{m}$ in the upper panel and (a-f) in the lower panel (a-i). Upper panel, Parkinson's disease (PD). a There is an amorphous deposit of aSyn (arrow) in the CCO-positive cytoplasm. $\mathbf{b}$ In the center of the aSyn-positive LB (arrow), the expression of CCO is negative. c The sausage-like Lewy body (LB) has a small rim, which is CCO-positive (arrow). $\mathbf{d}$ There are a few PaSynpositive granules (arrow) that are mildly TOM20-positive on the surface. e The neuron is filled with sausage-like LBs arrows, which are almost negative for TOM20. $\mathbf{f}$ In the neuron, the surface of the LB is mildly positive for TOM20. Lower panel, PLA2G6-associated neurodegeneration (PLAN) a There are some CCO-positive dots around the irregular aSyn-positive deposit (arrow). b aSyn-positive inclusion (arrow) contains some CCO-positive granules. c CCO expression is seen in only a part of the surface (arrows) of aSyn-positive LB. $\mathbf{d}$ Under the aSyn deposit, there are clear TOM20positive dots (arrow) in the cytoplasm. e In the neuronal cytoplasm, there are several PaSyn-positive granules (arrow), on which the surface is completely TOM20-positive. $\mathbf{f}$ PaSyn-positive granules are clustered to form a thin inclusion (arrow), on which the surface is positive for TOM20. $\mathbf{g}$ PaSynpositive granules with TOM2O-positive rim are clustered to form one round inclusion (arrow). $\mathbf{h} \mathbf{l n}$ the core of the LB, the immunoreaction of PaSyn and TOM20 are absent. In the halo of LB (arrow), PaSyn-positive granules with a TOM20-positive rim make a line. i PaSyn and TOM20-positive membrane-like structure covers the surface of LB (arrow) 
(Fig. 5b-f). The size of LBs in PLAN varied, as it does in PD.

\section{PaSyn -positive small inclusions colocalized with the mitochondrial membrane protein in PLAN}

To assess the relationship between $\alpha \operatorname{Syn} / \mathrm{P} \alpha \mathrm{Syn}$ deposits and mitochondria, we analyzed the neurons affected in PD and in PLAN using a double immunohistochemical assay. Double immunohistochemistry revealed amorphous deposits of $\alpha$ Syn in the CCOand TOM20-positive cytoplasm of neurons both in PD and in PLAN patients (PD; Fig. 6a, PLAN; Fig. 6d). As more $\alpha$ Syn accumulated in the neuronal cytoplasm, the CCO-immunoreactivity diminished (PD: Fig. 6b, c, PLAN: Fig. 6a-c). In PLAN neurons, small PoSyn-positive inclusions with a TOM20-positive edge were frequently found, but similar inclusions were rare in PD (PD: Fig. 6d-f, PLAN: Fig. 6e).

\section{Clustering of PaSyn-positive small inclusions to form a larger neuronal inclusion in PLAN}

To clarify the relationship between P $\alpha$ Syn-positive small inclusions and LBs, we analyzed the distribution of P $\alpha$ Syn-positive small inclusions in neurons affected in PLAN. In PLAN neurons, small P $\alpha$ Syn-positive inclusions with a TOM20-positive edge appeared to cluster, and formed a larger narrow (PLAN: Fig. 6f) or round (PLAN: Fig. 6g) inclusion. In some LBs, small PoSyn-positive inclusions with a TOM20positive edge appeared to cover the surface (PLAN: Fig. 6h), whereas the immunoreactivity of P $\alpha$ Syn was reduced in the center. P $\alpha$ Syn and TOM20-positive membranous structures also appeared to cover the surface of LBs (Fig. 6i).

\section{Quantitative analysis of LBs with TOM20 present on their surface}

To evaluate the relationship between LBs and mitochondria quantitatively, we counted the number of LBs with mitochondrial membrane protein (TOM20) present on their surface in PD and in PLAN. The number of LBs in each area is shown in Fig. 7. In all three investigated areas of the PD brains, most LBs were TOM20-negative on the surface. In the PLAN brain, most LBs were TOM20-positive on the surface. In all three investigated areas of the PLAN brain, more than half of the LBs were completely TOM20-positive. The surfaces of most LBs were TOM20-positive in PLAN and TOM20-negative in PD brains.

\section{Proportion of LB-bearing dopaminergic neurons and neuronal density}

To assess the survival rates of LB-bearing dopaminergic neurons, we estimated the neuronal density and the proportion of LB-harboring neurons in both PD and PLAN. The density of LB-bearing TH-positive neurons and total number of TH-positive neurons in substantia nigra and locus coeruleus are shown in Table 2. In the PD brains, the density of LB-bearing TH-positive neurons was low, because most LBs were found in the neuropil; this density was $1 \pm 1$ (mean \pm S.D.) in the substantia nigra and $2 \pm 2$ in the locus coeruleus (number per $1 \mathrm{~mm}^{2}$ ). In the PLAN brain, the density of LB-bearing neurons was 6 in the substantia nigra and 17 in the locus coeruleus, per $1 \mathrm{~mm}^{2}$. The density of TH-positive neurons in the substantia nigra

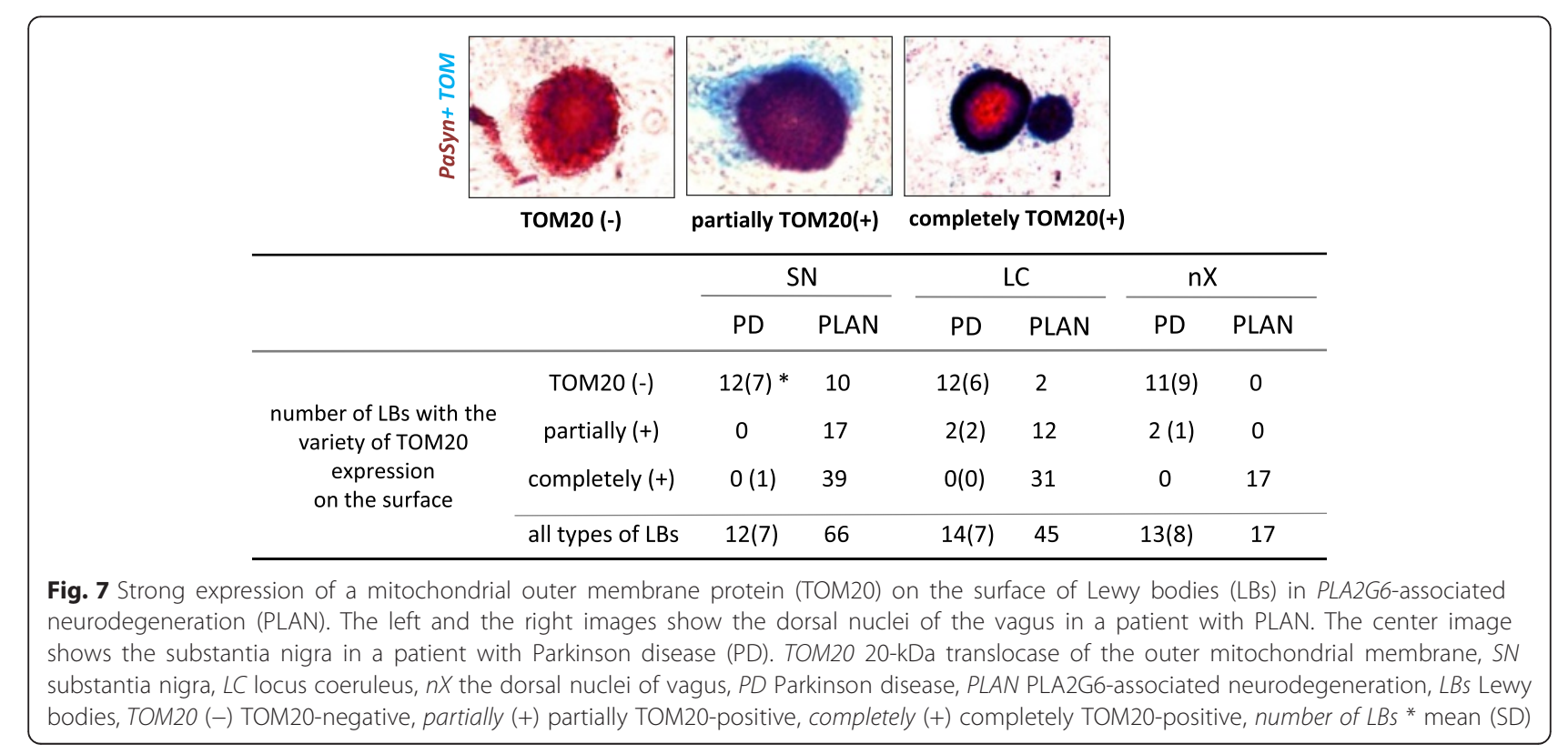


Table 2 The dopaminergic neuron density and the proportion of Lewy body-bearing neurons

\begin{tabular}{|c|c|c|c|c|}
\hline \multirow[t]{3}{*}{ Area } & & \multicolumn{3}{|c|}{ Density of dopaminergic neurons } \\
\hline & & Control & PD & PLAN \\
\hline & & $(n=5)$ & $(n=7)$ & $(n=1)$ \\
\hline \multirow[t]{2}{*}{ Substantia nigra } & LB harbouring neuron & 0 & $1(1)$ & 6 \\
\hline & total neuron & $84(14)$ & $13(6)$ & 17 \\
\hline \multirow[t]{2}{*}{ Locus coeruleus } & LB harbouring neuron & 0 & $1(3)$ & 17 \\
\hline & total neuron & $77(13)$ & $9(10)$ & 81 \\
\hline
\end{tabular}

TH tyrosine hydroxylase, Density of TH (+) neurons mean (SD) per $\mathrm{mm}^{2}$, control non-neurodegenerative disease, PD Parkinson disease, $P L A N$ PLA2G6-associated neurodegeneration, $L B$ Lewy body

was low, but that in the locus coeruleus was similar to that in the control brains, despite the prominent presence of LBs (Fig. 5e, f). The proportion of LB-bearing dopaminergic neurons was $6 / 17$ in the substantia nigra and $17 / 81$ in the locus coeruleus, respectively. Thus, a high proportion of LB-bearing neurons and preserved neuronal number were observed in PLAN.

\section{Preserved expression of $\mathrm{TH}$ in dopaminergic neurons, despite the presence of LBs}

To determine if LB influences the micro-environment in dopaminergic neurons, we estimated cytoplasmic TH expression of LB-bearing neurons. Both in PD and PLAN brains, most of the cytoplasmic $\mathrm{TH}$ expression in LB bearing neurons was preserved, both in substantia nigra and locus coeruleus (Table 3). Some LB-bearing neurons were $\mathrm{TH}$-negative. No relationship between cytoplasmic $\mathrm{TH}$ expression and LB presence was found.

\section{Discussion and conclusions}

In this study, we demonstrated elevated expression of $\alpha$ Syn both in cultured cells and in mice with PLA2G6 deficiency. Prominent accumulation of P $\alpha$ Syn in damaged mitochondria was shown in Pla2g6-KO mice, which constitute a model of PLA2G6-associated neurodegeneration (PLAN). In PLAN neurons, small P $\alpha$ Syn-positive granules and Lewy Bodies (LBs) were covered with mitochondrial membrane component on the surface. These findings suggest that $\alpha \operatorname{Syn} / \mathrm{P} \alpha \operatorname{Syn}$ associates with damaged mitochondria in PLAN.
Strong expression of $\alpha$ Syn was evident in Pla2g6-Kd neuroblastoma cells and KO mouse neurons, suggesting that endogenous $\alpha$ Syn was induced in neurons due to PLA2G6-deficiency. In Pla2g6-KO mice, surprisingly high levels of P $\alpha$ Syn expression were observed in neurons; this was never seen in the wild-type mice. P $\alpha$ Syn was detected on the surface of tiny granules, which had a staining pattern that was apparently different from that of the toxic strains of $\alpha$ Syn, including fibrils and ribbons $[6,33]$. Immunohistochemical and ultra-microscopic data suggest that P $\alpha$ Syn-positive and PAS-positive granules were abnormal mitochondria with degenerated inner membranes in $\mathrm{KO}$ mice, but not lysosomes or endoplasmic reticulum, as has been shown previously [4, 45].

Injury of the inner mitochondrial membrane leads to permeabilization of mitochondria $[2,32,50]$. In $\mathrm{KO}$ mice from young, many neuronal mitochondria with degenerated inner membrane were present, but neither autophagic degradation (mitophagy) nor ubiquitination of mitochondria were observed. Mitochondrial membrane permeabilization also induces apoptosis [32, 49]. More than 8 months after the appearance of mitochondria with degenerated inner membranes, due to PLA2G6 deficiency, neuronal loss was not significant in mice, suggesting that apoptosis did not occur to neurons. TOM20-positive granules were ubiquitin-negative in neurons and ubiquitin-positive in spheroids, suggesting ubiquitination of mitochondria occurs in degenerated axons, but not in neurons.

In aged $\mathrm{KO}$ mice, $\mathrm{P} \alpha \mathrm{Syn}$-positive granules became more degenerated morphologically, but the PaSyn expression

Table 3 The expression of tyrosine hydroxylase in dopaminergic neurons bearing Lewy bodies (LBs)

\begin{tabular}{|c|c|c|c|c|c|}
\hline & & \multicolumn{2}{|l|}{ SN } & \multicolumn{2}{|l|}{$\underline{\mathrm{LC}}$} \\
\hline & & PD & PLAN & PD & PLAN \\
\hline & & $(n=7)$ & $(n=1)$ & $(n=7)$ & $(n=1)$ \\
\hline \multirow{3}{*}{$\begin{array}{l}\text { Number of LB bearing neurons with the variety } \\
\text { of tyrosine hydroxylase expression }\end{array}$} & $\mathrm{TH}(+)$ & $3(2)^{*}$ & 34 & $2(4)$ & 19 \\
\hline & $\mathrm{TH}(-)$ & $1(1)$ & 3 & $1(1)$ & 0 \\
\hline & total number & $3(2)$ & 37 & $3(4)$ & 19 \\
\hline
\end{tabular}

$L B$ Lewy body, $S N$ substantia nigra, $L C$, locus coeruleus, $T H$ tyrosine hydroxylase, $T H(+)$ TH-positve neuron, $T H(-)$ TH-negative neuron, Number of neurons mean (SD) 


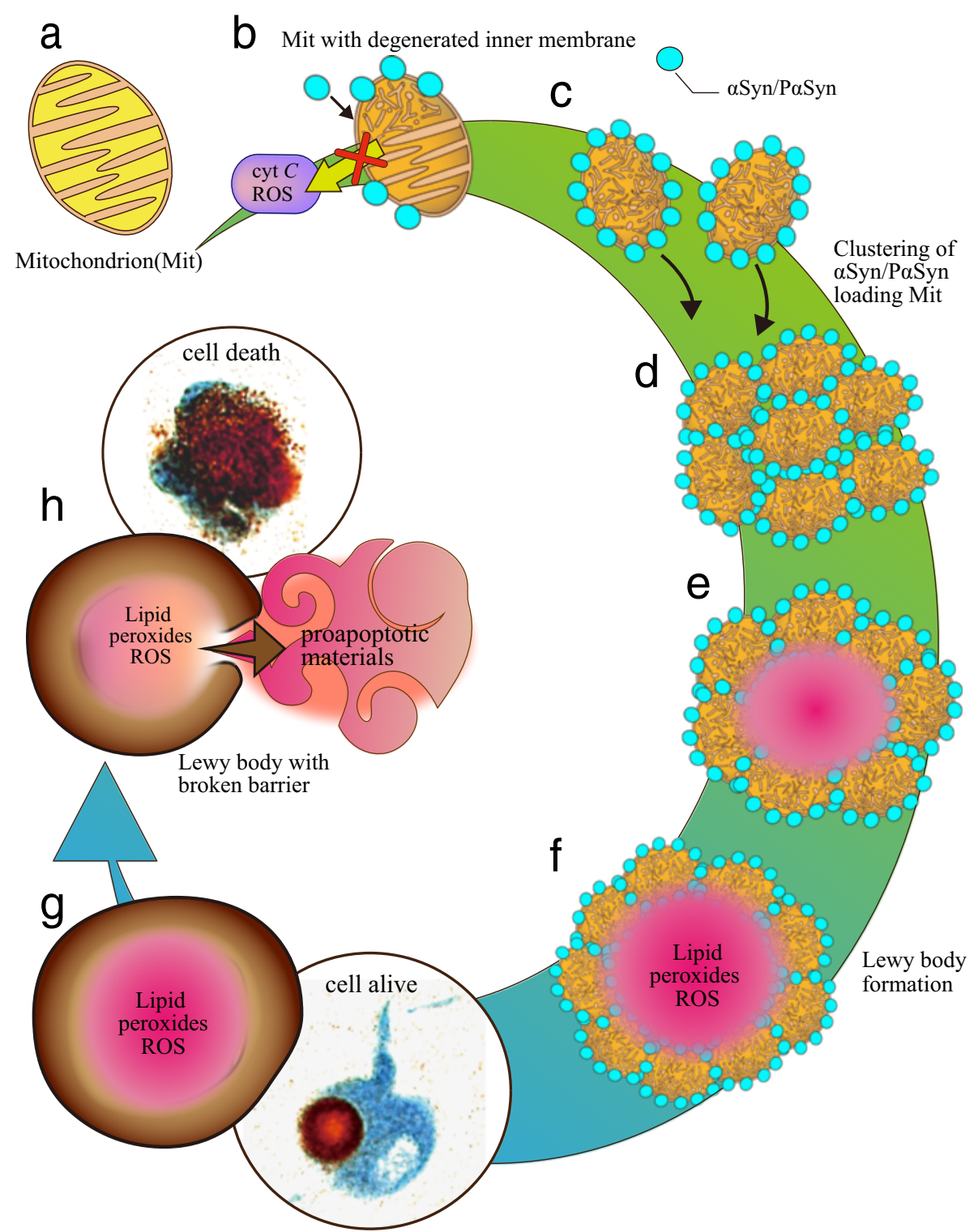

Fig. 8 The hypothetical scheme of the process of Lewy body formation in PLA2G6-associated neurodegeneration (PLAN). a Normal mitochondrion. b aSyn/PaSyn accumulates at the damaged mitochondrial membrane with PLA2G6 dysfunction. $\mathbf{c}$ High expression of aSyn/PaSyn stabilizes the mitochondrial membrane. $\mathbf{d}$ Due to the high affinity of aSyn for damaged mitochondrial membranes, mitochondria loaded with aSyn/PaSyn are clustered. e The cluster of the damaged mitochondria forms a small inclusion that contains toxic materials inside. $\mathbf{f}$ The small inclusion becomes large to be a Lewy body $(L B)$, which boundary is formed by aSyn/PaSyn and mitochondrial membrane components. $\mathbf{g}$ When the boundary, which is sufficiently strong to sequester toxic materials inside, the LB-bearing neuron survives for a long time. $\mathbf{h}$ When this boundary breaks down, pro-apoptotic materials are released into the neuron, leading to rapid neuronal death. The image of neurons double-stained with immunohistochemistry for PaSyn (in brown) and tyrosine hydroxylase (in blue) is taken from the locus coeruleus of a patient with PLAN in (g), and a patient with PD in (h)

level was maintained. $\alpha$ Syn/P $\alpha$ Syn seems to have a strong affinity for the mitochondrial membrane [37, 52]. However, considering the different localization of P $\alpha$ Syn and ubiquitin in axons, P $\alpha$ Syn seems to disappear from mitochondria before mitochondrial ubiquitination, following membrane permeabilization. The elevated expression of $\alpha \operatorname{Syn} / \mathrm{P} \alpha \operatorname{Syn}$ in mitochondria may be related to membrane stabilization [40].
In the substantia nigra of $26 \mathrm{~S}$ proteasome depleted mice, neurodegeneration is accompanied by neuronal inclusions similar to Pale bodies [30], the precursors of LB [46]. The inclusions contain mainly abnormal mitochondria [30], suggesting that Pale body or LB might be associated with abnormal mitochondria with bad quality control owing to dysfunction of ubiquitin proteasome system. As previously reported, LB formation is prominent in 
PLAN [15, 31, 36]. Considering the severity of mitochondrial degeneration in Pla2g6-KO mice, the association of Pale bodies or LBs with mitochondria would be strongly expected in PLAN.

Our double immunohistochemical data in PLAN revealed that the surface of PaSyn-positive small inclusions were TOM20-positive and CCO-negative, which is the same expression pattern of granules seen in Pla2g6$\mathrm{KO}$ mouse neurons. $\alpha \mathrm{Syn} / \mathrm{P} \alpha \mathrm{Syn}$ appears to have a strong affinity for mitochondrial membrane components both in Pla2g6-KO mice and in the PLAN case investigated here. Moreover, the small inclusions appeared to cluster into a large inclusion, possibly due to the strong affinity of $\alpha$ Syn for the mitochondrial membrane.

LB extracts from PD brains have been reported to trigger $\alpha$ Syn pathology and motor deficits in mice and monkeys, which suggests that LBs contain neurotoxic materials [35]. However, the presence of LBs in dopaminergic neurons was not associated with the low cytoplasmic expression of $\mathrm{TH}$, either in the autopsied PLAN or PD brains studied here, although this occurs early in PD [21]. This suggests that the presence of LB would not necessarily convey negative effects on the microenvironment of neurons, in spite of the neurotoxic materials inside the LB.

A certain proportion of LB-bearing neurons is reported to occur in the substantia nigra $(3.6 \%$ on average), and this does not correlate with the symptoms or disease duration [14, 18]. In our case of PLAN, a high proportion of dopaminergic neurons harbor LBs, suggesting the long-term survival of these neurons. In particular, the neurons were preserved well in the locus coeruleus, despite the presence of multiple LBs. This suggests that LBs in PLAN have a low toxicity. As $\alpha$ Syn $/ \mathrm{P} \alpha$ Syn-loading mitochondria with degenerated inner membrane did not lead to neuronal death in mice, high concentrations of mitochondrial membrane components on the surface of LBs may be associated with the low toxicity of LBs in PLAN through the stabilization of the mitochondrial lipid membrane [7, 29].

We illustrate this hypothesis in Fig. 8. Because of PLA2G6 dysfunction, the mitochondrial inner membrane is damaged early on. Strong affinity of $\alpha \mathrm{Syn} / \mathrm{P} \alpha \mathrm{Syn}$ for damaged mitochondrial membrane induces Syn/ P $\alpha$ Syn to accumulate on the membrane. Elevated expression of $\alpha \operatorname{Syn} / \mathrm{P} \alpha$ Syn stabilizes the mitochondrial membrane and prevents membrane permeabilization. The strong affinity of $\alpha$ Syn for damaged mitochondrial membranes also causes clustering of the damaged mitochondria to form LB. The outer structure, which is comprised of a mitochondrial membrane component, functions as a barrier and allows LB-bearing neurons to survive for a long time. When the barrier breaks down, pro-apoptotic materials, which are elevated in PLA2G6 deficiency [20], are released into the cytoplasm, followed by rapid cell death.

In conclusion, $\alpha$ Syn $/ \mathrm{P} \alpha$ Syn accumulation in damaged mitochondria of Pla2g6-KO mice and LBs in PLAN indicates a common pathological mechanism, which includes the strong affinity of $\alpha$ Syn for damaged mitochondrial membranes. PLAN patients with late onset tend to show prominent $\alpha$ Syn accumulation in neurons [31]. Endogenous $\alpha$ Syn could be neuroprotective by stabilizing mitochondrial membranes in neurodegenerative diseases, such as PLAN and possibly even in PD, which may indicate a new target for the treatment of $\alpha$ synucleinopathy.

\section{Competing interests}

The authors declare that they have no competing interests.

\section{Authors' contributions}

HSA contributed the study design, data acquisition, analysis and manuscript preparation. GB and KS contributed to data acquisition, analysis and manuscript preparation. SK contributed to study design, data analysis and manuscript preparation. YR and MY contributed to data analysis and manuscript preparation. HF, YT and SS contributed to manuscript preparation. HM contributed the study design and manuscript preparation. All authors have read and approved the final manuscript.

\section{Acknowledgements}

This study was supported in part by a Grant-in-Aid for Scientific Research (C) from the Japan Society for the Promotion of Science, JSPS (H.SA. G.B.), Scientific Research on Innovative Areas from MEXT (H.M.), Brain Mapping by Integrated Neurotechnologies for Disease Studies (Brain/MINDS) (H.M.), Practical Research Project for Rare/Intractable Disease from Japan Agency for Medical Research and Development, AMED (S.K., H.M.), and a Budget for Promoting Priority Measures in Discretionary Funds of the President in Tottori University (S.K.). The authors would like to thank H. Hayakawa for his expert assistance with biological analysis, K. Inoue for her expert assistance with pathological analysis, A. Sone for technical assistance, and R. Yoshida for fantastic illustrations.

\section{Author details}

'Department of Neurology, Graduate School of Medicine, Osaka University, 2-2 Yamadaoka, Suita 565-0871, Japan. ${ }^{2}$ Department of Medical Genetics, Graduate School of Medicine, Osaka University, 2-2 Yamadaoka, Suita 565-0871, Japan. ${ }^{3}$ Division of Neuropathology, Department of Brain and Neurosciences, Tottori University Faculty of Medicine, 86 Nishi-machi, Yonago 683-8504, Japan. ${ }^{4}$ Department of Neurology, Nagoya University Graduate School of Medicine, 65 Tsurumai-cho, Showa-ku, Nagoya 466-8550, Japan. ${ }^{5}$ Institute for Medical Science of Aging, Aichi Medical University, 9 Yazakokarimata, Nagakute 480-1195, Japan. ${ }^{6}$ Department of Neurology, National Hospital Organization Toneyama National Hospital, 5-1-1 Toneyama, Toyonaka 560-8552, Japan. ${ }^{7}$ Research Institute Osaka Medical Center for Cancer and Cardiovascular Diseases, 1-3-3 Nakamichi, Osaka 537-0025, Japan.

Received: 4 February 2016 Accepted: 10 March 2016

Published online: 30 March 2016

References

1. Araki K, Yagi N, Ikemoto Y, Choong CJ, Hayakawa H, Beck G, Sumi H, Fujimura H, Moriwaki T, Nagai Y, Goto Y, Mochizuki H. Synchrotron FTIR micro-spectroscopy for structural analysis of Lewy bodies in the brain of Parkinson's disease patients. Sci Rep. 2015;5:17625.

2. Ashrafi G, Schwarz TL. The pathways of mitophagy for quality control and clearance of mitochondria. Cell Death Differ. 2013;20:31-42.

3. Beck G, Shinzawa K, Hayakawa H, Baba K, Yasuda T, Sumi-Akamaru H, Tsujimoto Y, Mochizuki H. Deficiency of Calcium-Independent Phospholipase A2 Beta Induces Brain Iron Accumulation through 
Upregulation of Divalent Metal Transporter 1. PLoS One. 2015;10(10), e0141629.

4. Beck G, Sugiura Y, Shinzawa K, Kato S, Setou M, Tsujimoto Y, Sakoda S, Sumi-Akamaru $\mathrm{H}$. Neuroaxonal dystrophy in calcium-independent phospholipase $A_{2} B$ deficiency results from insufficient remodeling and degeneration of mitochondrial and presynaptic membranes. J Neurosci. 2011:31:11411-20

5. Bendor JT, Logan TP, Edwards RH. The Function of a-Synuclein. Neuron. 2013;79:1044-66.

6. Bousset L, Pieri L, Ruiz-Arlandis G, Gath J, Jensen PH, Habenstein B, Madiona K, Olieric V, Böckmann A, Meier BH, Melki R. Structural and functional characterization of two alpha-synuclein strains. Nat Commun. 2013;4:2575.

7. Braun AR, Lacy MM, Ducas VC, Rhoades E, Sachs JN. a-Synuclein-induced membrane remodeling is driven by binding affinity, partition depth, and interleaflet order asymmetry. J Am Chem Soc. 2014;136:9962-72.

8. Burke JE, Dennis EA. Phospholipase A2 structure/function, mechanism, and signaling. J Lipid Res. 2009;50(Suppl):S237-42.

9. Burré J, Sharma M, Tsetsenis T, Buchman V, Etherton MR, Südhof TC. Alphasynuclein promotes SNARE-complex assembly in vivo and in vitro. Science. 2010;329:1663-7.

10. Cheon Y, Kim HW, Igarashi M, Modi HR, Chang L, Ma K, Greenstein D, Wohltmann M, Turk J, Rapoport SI, Taha AY. Disturbed brain phospholipid and docosahexaenoic acid metabolism in calcium-independent phospholipase $\mathrm{A}_{2}$-VIA (iPLA ${ }_{2} \beta$ )-knockout mice. Biochim Biophys Acta. 2012; 1821:1278-86.

11. Cowen D, Olmstead EV. Infantile neuroaxonal dystrophy. J Neuropathol Exp Neurol. 1963;22:175-236.

12. Ellis CE, Murphy EJ, Mitchell DC, Golovko MY, Scaglia F, Barceló-Coblijn GC, Nussbaum RL. Mitochondrial lipid abnormality and electron transport chain impairment in mice lacking alpha-synuclein. Mol Cell Biol. 2005;25:10190-201.

13. Glynn P. Neuronal phospholipid deacylation is essential for axonal and synaptic integrity. Biochim Biophys Acta. 2013;1831:633-41.

14. Greffard S, Verny M, Bonnet AM, Seilhean D, Hauw JJ, Duyckaerts C. A stable proportion of Lewy body bearing neurons in the substantia nigra suggests a model in which the Lewy body causes neuronal death. Neurobiol Aging. 2010:31:99-103.

15. Gregory A, Westaway SK, Holm IE, Kotzbauer PT, Hogarth P, Sonek S, et al. Neurodegeneration associated with genetic defects in phspholipase $A_{2}$. Neurology. 2008;71:1402-9.

16. Guardia-Laguarta C, Area-Gomez E, Rüb C, Liu Y, Magrané J, Becker D, Voos W, Schon EA, Przedborski S. a-Synuclein is localized to mitochondria-associated ER membranes. J Neurosci. 2014;34:249-59.

17. Hedley-Whyte ET, Gilles FH, Uzman BG. Infantile neuroaxonal dystrophy. A disease characterized by altered terminal axons and synaptic endings. Neurology. 1968;18:891-6.

18. Jellinger KA. Neuropathology of sporadic Parkinson's disease: Evaluation and changes of concepts. Mov Disord. 2012;27:8-30.

19. Khateeb S, Flusser H, Ofir R, Shelef I, Narkis G, Vardi G, Shorer Z, Levy R, Galil A, Elbedour K, Birk OS. PLA2G6 mutation underlies infantile neuroaxonal dystrophy. Am J Hum Genet. 2006;79:942-8.

20. Kinghorn KJ, Castillo-Quan Jl, Bartolome F, Angelova PR, Li L, Pope S, Cochemé HM, Khan S, Asghari S, Bhatia KP, Hardy J, Abramov AY, Partridge L. Loss of PLA2G6 leads to elevated mitochondrial lipid peroxidation and mitochondrial dysfunction. Brain. 2015;138(Pt 7):1801-16.

21. Kordower JH, Olanow CW, Dodiya HB, Chu Y, Beach TG, Adler CH, Halliday GM, Bartus RT. Disease duration and the integrity of the nigrostriatal system in Parkinson's disease. Brain. 2013;136(Pt 8):2419-31.

22. Lai Y, Kim S, Varkey J, Lou X, Song JK, Diao J, Langen R, Shin YK. Nonaggregated a-synuclein influences SNARE-dependent vesicle docking via membrane binding. Biochemistry. 2014;53:3889-96.

23. Malik I, Turk J, Mancuso DJ, Montier L, Wohltmann M, Wozniak DF, Schmidt RE, Gross RW, Kotzbauer PT. Disrupted membrane homeostasis and accumulation of ubiquitinated proteins in a mouse model of infantile neuroaxonal dystrophy caused by PLA2G6 mutations. Am J Pathol. 2008; 172:406-16.

24. Marland JR, Hasel P, Bonnycastle K, Cousin MA. Mitochondrial Calcium Uptake Modulates Synaptic Vesicle Endocytosis in Central Nerve Terminals. J Biol Chem. 2016;291:2080-6.

25. Monteiro JP, Oliveira PJ, Jurado AS. Mitochondrial membrane lipid remodeling in pathophysiology: a new target for diet and therapeutic interventions. Prog Lipid Res. 2013;52:513-28.
26. Morgan NV, Westaway SK, Morton JE, Gregory A, Gissen P, Sonek S, et al. PLA2G6, encoding a phospholipase A2, is mutated in neurodegenerative disorders with high brain iron. Nat Genet. 2006;38:752-4.

27. Morrison K, Witte K, Mayers JR, Schuh AL, Audhya A. Roles of acidic phospholipids and nucleotides in regulating membrane binding and activity of a calcium-independent phospholipase A2 isoform. J Biol Chem. 2012;287:38824-34

28. Murakami M, Taketomi Y, Miki Y, Sato H, Hirabayashi T, Yamamoto K. Recent progress in phospholipase A2 research: from cells to animals to humans. Prog Lipid Res. 2011;50:152-92.

29. Ouberai MM, Wang J, Swann MJ, Galvagnion C, Guilliams T, Dobson CM, Welland ME. $a$-Synuclein senses lipid packing defects and induces lateral expansion of lipids leading to membrane remodeling. J Biol Chem. 2013; 288:20883-95.

30. Paine SM, Anderson G, Bedford K, Lawler K, Mayer RJ, Lowe J, Bedford L. Pale body-like inclusion formation and neurodegeneration following depletion of 265 proteasomes in mouse brain neurones are independent of a-synuclein. PLoS One. 2013;8, e54711.

31. Paisán-Ruiz C, Li A, Schneider SA, Holton JL, Johnson R, Kidd D, Chataway J, Bhatia KP, Lees AJ, Hardy J, Revesz T, Houlden H. Widespread Lewy body and tau accumulation in childhood and adult onset dystonia-parkinsonism cases with PLA2G6 mutations. Neurobiol Aging. 2012;33:814-23.

32. Paradies $G$, Paradies V, De Benedictis V, Ruggiero FM, Petrosillo G. Functional role of cardiolipin in mitochondrial bioenergetics. Biochim Biophys Acta. 2014; 1837:408-17.

33. Peelaerts W, Bousset L, Van der Perren A, Moskalyuk A, Pulizzi R, Giugliano M, Van den Haute C, Melki R, Baekelandt V. a-Synuclein strains cause distinct synucleinopathies after local and systemic administration. Nature. 2015; 522(7556):340-4

34. Polymeropoulos MH, Lavedan C, Leroy E, Ide SE, Dehejia A, Dutra A, et al. Mutation in the alpha-synuclein gene identified in families with Parkinson's disease. Science. 1997;276:2045-7.

35. Recasens A, Dehay B, Bové J, Carballo-Carbajal I, Dovero S, Pérez-Villalba A, Fernagut PO, Blesa J, Parent A, Perier C, Fariñas I, Obeso JA, Bezard E, Vila M. Lewy body extracts from Parkinson disease brains trigger a-synuclein pathology and neurodegeneration in mice and monkeys. Ann Neurol. 2014; 75:351-62.

36. Riku Y, Ikeuchi T, Yoshino H, Mimuro M, Mano K, Goto Y, Hattori N, Sobue G, Yoshida M. Extensive aggregation of a-synuclein and tau in juvenile-onset neuroaxonal dystrophy: an autopsied individual with a novel mutation in the PLA2G6 gene-splicing site. Acta Neuropathol Commun. 2013;1:12.

37. Robotta M, Gerding HR, Vogel A, Hauser K, Schildknecht S, Karreman C, Leist M, Subramaniam V, Drescher M. Alpha-synuclein binds to the inner membrane of mitochondria in an a-helical conformation. Chembiochem. 2014;15:2499-502.

38. Ross OA, Braithwaite AT, Skipper LM, Kachergus J, Hulihan MM, Middleton FA, et al. Genomic investigation of alpha-synuclein multiplication and parkinsonism. Ann Neurol. 2008;63:743-50.

39. Sengoku R, Saito Y, Ikemura M, Hatsuta H, Sakiyama Y, Kanemaru K, Arai T, Sawabe M, Tanaka N, Mochizuki H, Inoue K, Murayama S. Incidence and extent of Lewy body-related alpha-synucleinopathy in aging human olfactory bulb. J Neuropathol Exp Neurol. 2008;67:1072-83.

40. Shen J, Du T, Wang X, Duan C, Gao G, Zhang J, Lu L, Yang H. a-Synuclein amino terminus regulates mitochondrial membrane permeability. Brain Res. 2014;1591:14-26.

41. Shinzawa K, Sumi H, Ikawa M, Matsuoka Y, Okabe M, Sakoda S, Tsujimoto Y. Neuroaxonal dystrophy caused by group VIA phospholipase A2 deficiency in mice: a model of human neurodegenerative disease. J Neurosci. 2008;28: 2212-20.

42. Shvadchak W, Yushchenko DA, Pievo R, Jovin TM. The mode of a-synuclein binding to membranes depends on lipid composition and lipid to protein ratio. FEBS Lett. 2011:585:3513-9.

43. Snead D, Eliezer D. Alpha-synuclein function and dysfunction on cellular membranes. Exp Neurobiol. 2014;23:292-313.

44. Sumi H, Nagano S, Fujimura H, Kato S, Sakoda S. Inverse correlation between the formation of mitochondria-derived vacuoles and Lewy-bodylike hyaline inclusions in G93A superoxide-dismutase-transgenic mice. Acta Neuropathol. 2006;112:52-63.

45. Sumi-Akamaru H, Beck G, Kato S, Mochizuki H. Neuroaxonal dystrophy in PLA2G6 knockout mice. Symposium: Neurodegeneration with Brain Iron Accumulation. Neuropathology. 2015;35:289-302. 
46. Wakabayashi K, Tanji K, Odagiri S, Miki Y, Mori F, Takahashi H. The Lewy body in Parkinson's disease and related neurodegenerative disorders. Mol Neurobiol. 2013;47:495-508.

47. Wilson RS, Nag S, Boyle PA, Hizel LP, Yu L, Buchman AS, Shah RC, Schneider JA, Arnold SE, Bennett DA. Brainstem aminergic nuclei and late-life depressive symptoms. JAMA Psychiatry. 2013;70:1320-8.

48. Yamada M, Iwatsubo T, Mizuno Y, Mochizuki H. Overexpression of asynuclein in rat substantia nigra results in loss of dopaminergic neurons, phosphorylation of a-synuclein and activation of caspase-9: resemblance to pathogenetic changes in Parkinson's disease. J Neurochem. 2004;91: 451-61.

49. Yin H, Zhu M. Free radical oxidation of cardiolipin: chemical mechanisms, detection and implication in apoptosis, mitochondrial dysfunction and human diseases. Free Radic Res. 2012;46:959-74.

50. Youle RJ, Narendra DP. Mechanisms of mitophagy. Nat Rev Mol Cell Biol. 2011;12:9-14

51. Zhao Z, Zhang X, Zhao C, Choi J, Shi J, Song K, Turk J, Ma ZA. Protection of pancreatic beta-cells by group VIA phospholipase A (2)-mediated repair of mitochondrial membrane peroxidation. Endocrinology. 2010; 151:3038-48.

52. Zigoneanu IG, Yang YJ, Krois AS, Haque E, Pielak GJ. Interaction of asynuclein with vesicles that mimic mitochondrial membranes. Biochim Biophys Acta. 2012;1818:512-9.

\section{Submit your next manuscript to BioMed Central} and we will help you at every step:

- We accept pre-submission inquiries

- Our selector tool helps you to find the most relevant journal

- We provide round the clock customer support

- Convenient online submission

- Thorough peer review

- Inclusion in PubMed and all major indexing services

- Maximum visibility for your research

Submit your manuscript at www.biomedcentral.com/submit 